\title{
Application of quantitative analysis of contrast-enhanced ultrasonography in the evaluation of acute radiation-induced liver damage
}

\author{
TIAN-YOU XIN ${ }^{1}$, JUN FENG $^{1}$, SHU-BO CHEN $^{2}$, XIAN CHEN $^{1}$, \\ YA-YUN GUO ${ }^{1}$, PING SUN ${ }^{3}$ and YING-ZHEN CHEN ${ }^{1}$ \\ Departments of ${ }^{1}$ Ultrasound, ${ }^{2}$ Oncology and ${ }^{3}$ Pathology, The Second Hospital of Wuxi \\ Affiliated to Nanjing Medical University, Wuxi, Jiangsu 214002, P.R. China
}

Received May 8, 2019; Accepted January 10, 2020

DOI: $10.3892 /$ etm.2020.8541

\begin{abstract}
The aim of the present study was to evaluate the microcirculation perfusion in patients with acute radiation-induced liver damage (RILD) and explore the feasibility of non-invasive evaluation of RILD using quantitative analysis of contrast-enhanced ultrasound (CEUS). Patients who successfully underwent three-dimensional conformal intensity-modulated radiotherapy for abdominal tumors were selected. CEUS was performed on the liver prior to and 2,3 and 4 weeks after exposure, and the time-intensity curve (TIC) was obtained by quantitative analysis of CEUS. The time to peak (TTP), gradient (Grad) and area under the curve (AUC) were analyzed offline. The Grad of the CEUS TIC was decreased and TTP increased with the prolongation of the irradiation duration, with statistically significant differences between the values in the 2-, 3- and 4-week groups vs. those prior to exposure $(\mathrm{P}<0.05)$, as well as among the values of the 2 -, 3- and 4-week groups $(\mathrm{P}<0.05)$. Following irradiation, the AUC decreased gradually in the 2- and 3-week groups and increased in the 4-week group, with statistically significant differences compared with the AUC prior to irradiation $(\mathrm{P}<0.05)$. The quantitative analysis parameters of CEUS may be important reference parameters for the early diagnosis of acute RILD.
\end{abstract}

\section{Introduction}

Radiation-induced liver damage (RILD) refers to a series of pathophysiological changes that occur as a result of liver cell damage caused by exposure of the liver to a certain dose of

Correspondence to: Dr Jun Feng, Department of Ultrasound, The Second Hospital of Wuxi Affiliated to Nanjing Medical University, 68 Zhongshan Road, Wuxi, Jiangsu 214002, P.R. China

E-mail: wxfengjun@163.com

Key words: contrast medium, radiation-induced liver damage, ultrasonography, rays, quantitative analysis, time-intensity curve radiation (1). RILD is a common complication of radiotherapy for abdominal tumors. In most cases, early acute RILD may be repaired. However, if clinical symptomatic treatment is not administered, it may continue to develop into subacute RILD, eventually leading to liver failure with a high rate of mortality (2). Therefore, early detection and diagnosis of acute RILD are of great significance for restorative treatment of the liver.

Several studies have explored effective ways to diagnose and treat early-stage RILD, including the establishment of animal models of RILD and investigation of/using certain imaging methods. However, previous imaging studies using modalities including CT, MRI or single-photon emission CT, focused mainly on the discovery of morphological changes associated with RILD and were limited by the duration of the disease, and accordingly, their clinical application is currently limited $(3,4)$. Previous experimental studies on RILD in animals have demonstrated that contrast-enhanced ultrasound (CEUS) is able to directly reflect changes in the circulating dynamics of microvessels in early-stage RILD (5). The aim of the present study was therefore to assess the performance of CEUS in the quantitative evaluation of microcirculation changes in the liver parenchyma of patients with acute RILD, so as to explore effective diagnostic parameters.

\section{Materials and methods}

Participants. Patients who had undergone radiotherapy for the treatment of abdominal malignant tumors at the Second Hospital of Wuxi Affiliated to Nanjing Medical University (Wuxi, China) between August 2015 and August 2018 were recruited for the present study. A total of 32 patients were selected, including 21 males and 11 females aged 52-86 years (mean age, 68.8 \pm 10.6 years). Five cases of pancreatic head carcinoma, 7 of pancreatic body and tail tumor, 8 of gastric cancer following radiotherapy and 12 of abdominal metastatic lymph node treated with radiotherapy were enrolled in the present study. The tumor diameters were $3-6 \mathrm{~cm}$ and pancreatic cancer stages were IB-IIB (6). The exclusion criteria were as follows: i) Imaging examination prior to radiotherapy 

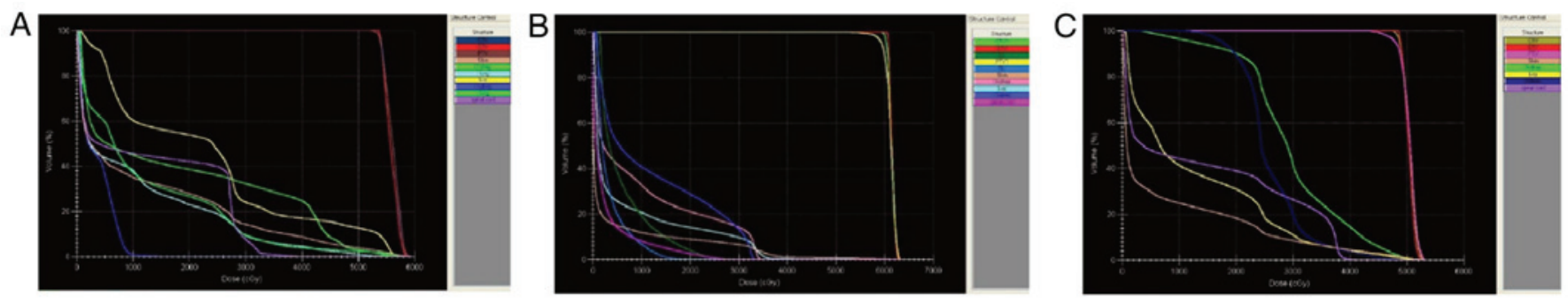

Figure 1. DVHs of three different patients. X-axis, total irradiation dose; Y-axis, organ exposure volume. The key chart on the right denotes different organs with varying colors and the yellow line refers to the DVH of the liver after exposure. (A) Radiotherapy for pancreatic head carcinoma. According to the DVH diagram, the volume of liver irradiation at the radiation dose of 2,500 cGy was $\sim 48 \%$. (B) Radiotherapy for left abdominal lymph node metastasis. According to the DVH diagram, the volume of liver irradiation at the radiation dose of 2,500 cGy was $\sim 12 \%$. (C) Radiotherapy for pancreatic tail tumor. According to the DVH diagram, the volume of liver irradiation at the radiation dose of 2,500 cGy was $\sim 18 \%$. DVH, dose-volume histogram; GTV, gross tumor volume; CTV, clinical target volume; PTV, planning target volume.

indicated diffuse liver lesions or benign or malignant occupying lesions in the liver; and ii) serological examination prior to radiotherapy suggested abnormal liver function.

The grouping criteria were as follows: According to the time of radiotherapy received for the treatment of malignant abdominal tumors, the patients were divided into 4 groups: i) Prior to radiotherapy; ii) 2 weeks after radiotherapy; iii) 3 weeks after radiotherapy; and iv) 4 weeks after radiotherapy.

Instruments and methods. The following instruments were used in the present study: A Primus linear accelerator (6MV-X; Siemens AG) and a LOGIQ E9 ultrasound instrument (GE Healthcare) with a low mechanical index enhancement mode, time-intensity curve (TIC) analysis software and convex array probe (frequency, 1-5 MHz). The contrast medium was SonoVue (Bracco Imaging SpA).

A CT simulator was used for positioning and an XIO4.80 treatment planning system (Elekta Instrument $\mathrm{AB}$ ) was used to delineate the target areas (gross tumor volume, clinical target volume and planning target volume) and design the exposure field. The entire liver was delineated according to the endangered organs, followed by exposure of 4-5 fields to conformal radiotherapy, in which the dose was divided conventionally, with an exposure dose rate of $200 \mathrm{cGy} / \mathrm{min}$.

The exposure doses of the liver were evaluated by a dose-volume histogram (Fig. 1) and the areas with exposure doses of 2,400-2,600 cGy/25-30 f were selected for ultrasonography, according to the isodose curve (Fig. 2).

Two-dimensional and color Doppler ultrasound was used to examine the liver prior to CEUS to observe the liver parenchyma and intrahepatic vessels. According to the isodose curve, suitable areas in the hepatic parenchyma were selected, avoiding the large blood vessels, and CEUS was performed after fixing the section. A bolus of SonoVue suspension $(2.4 \mathrm{ml})$ was injected via the elbow vein using a $16 \mathrm{G}$ intravenous indwelling needle, following which $5 \mathrm{ml}$ normal saline was injected. The contrast enhancement mode was turned on and the timer was triggered at the time of contrast medium injection. The dynamic data were acquired over $180 \mathrm{sec}$ and stored in digital imaging and communications in medicine (DICOM) format for offline analysis.

Data from all patients were analyzed using the same sonographer, who has $>15$ years of experience in contrast-enhanced ultrasound. A circular region of interest (ROI; diameter, $5 \mathrm{~mm}$ ) was selected in the preselected parenchyma region of the isodose curve. For each patient, the incision section for CEUS was kept consistent as much as possible. The TIC of the liver parenchyma was obtained using the software complementary to the instrument. The time until the appearance of the first contrast agent microbubble in the liver was defined as the arrival time, and the time-point $160 \mathrm{sec}$ after that as the end time, during which the hepatic blood perfusion was analyzed. In addition, the time to peak (TTP), gradient (Grad), and area under the curve (AUC) of the contrast in the liver parenchyma of the ROI were analyzed to determine the hemodynamic changes in the liver.

Statistical analysis. Data were statistically analyzed using SPSS 20.0 software (IBM Corp.). Measurement data following a normal distribution are expressed as the mean \pm standard deviation. The changes in the three indicators at each time-point were compared using single-factor repeated-measures analysis of variance and the changes prior to and after the exposure were compared using paired t-tests.

\section{Results}

General information. The present study included 32 patients that had undergone radiotherapy for abdominal tumors, conformed to the inclusion criteria and had stage IB-IIB pancreatic cancer. CEUS was performed on the liver of each patient prior to, as well as 2, 3 and 4 weeks after the exposure. The complete information obtained with CEUS was stored in DICOM. The TTP, Grad and AUC were analyzed and expressed by the TIC, which was obtained from the quantitative analysis of CEUS, so as to explore potential diagnostic parameters for RILD.

Ultrasonography results. CEUS was successfully performed on all patients. The TIC obtained by quantitative analysis is presented in Fig. 3, with the yellow curve representing the perfusion curve of the contrast in the liver parenchyma. Under normal liver tissue conditions, liver parenchyma enhancement caused by hepatic artery alone was performed for 10-20 sec after the contrast agent was injected into the peripheral vein for $10-15 \mathrm{sec}$. Subsequently, the portal vein phase was initiated, which occurred for 2 min after the contrast agent was injected. When contrast microbubbles began to clear from the liver parenchyma, it entered the delayed phase, which occurred from 4-6 min after the injection of SonoVue. Hence, in the present study, the TIC 


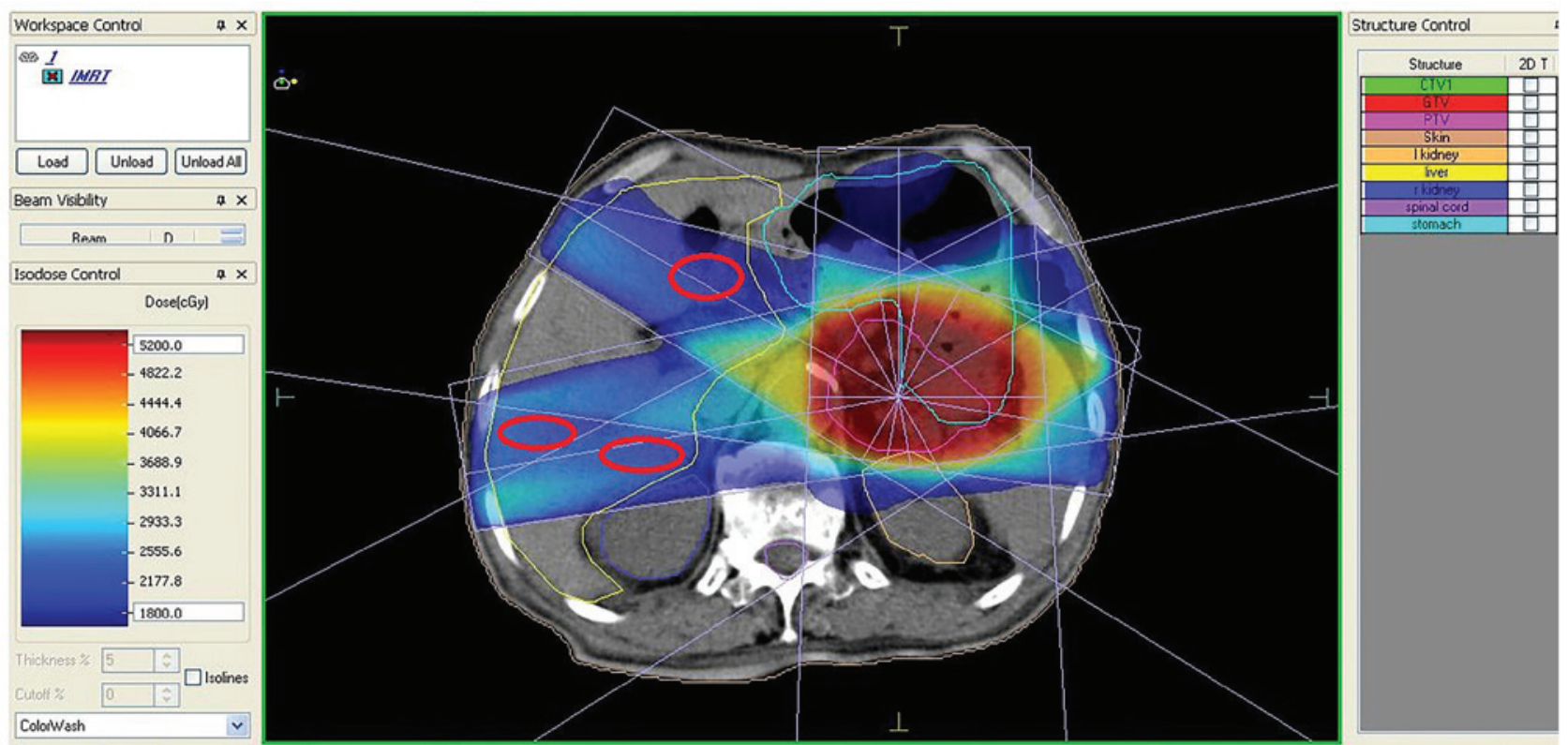

Figure 2. Isodose schematic diagram. The area with an exposure dose of 2,400-2,600 cGy/25-30 $\mathrm{f}$ in the liver (red circles) was selected as a detection point for contrast-enhanced ultrasound, according to the color in the legend on the left. GTV, gross tumor volume; CTV, clinical target volume; PTV, planning target volume.

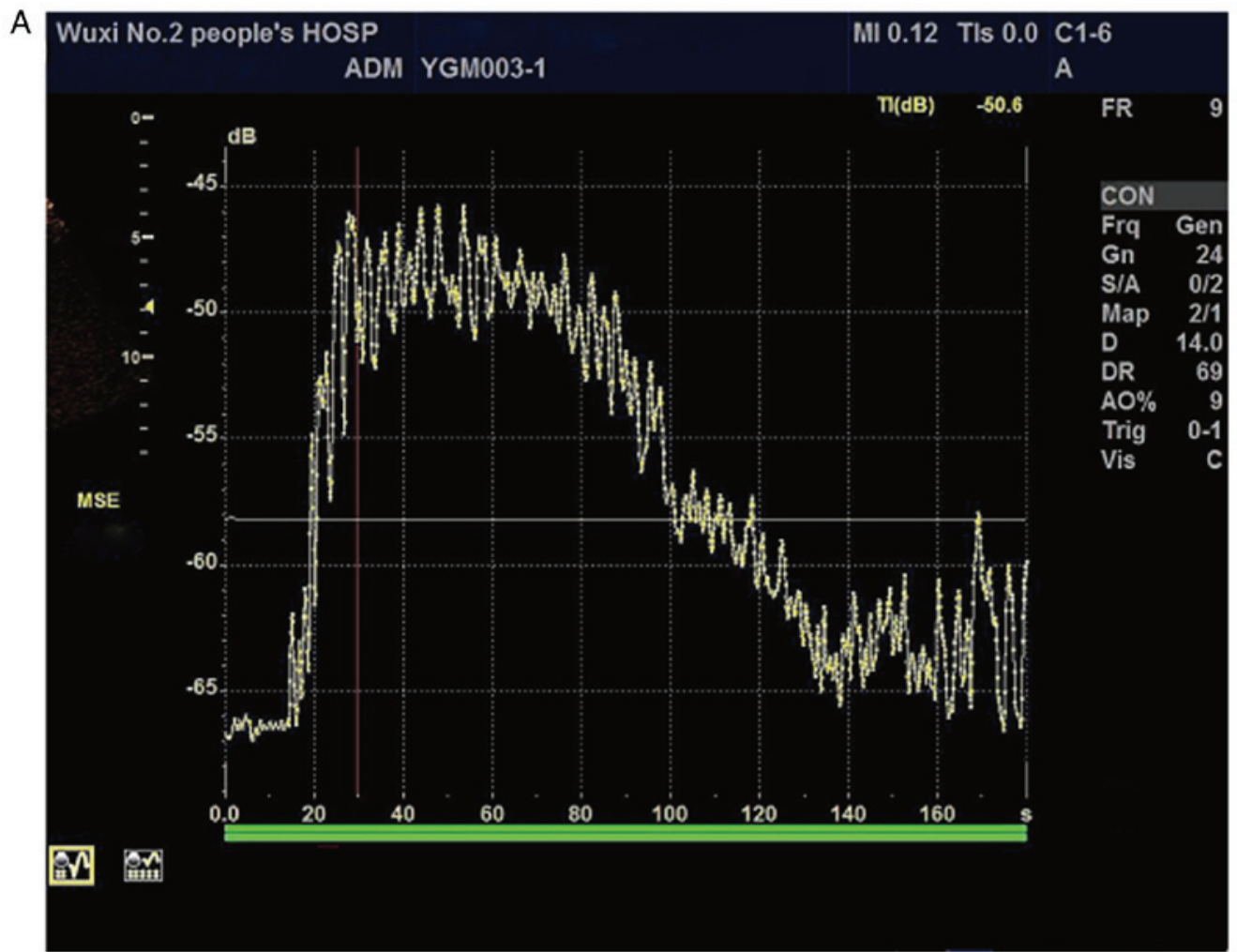

Figure 3. Images of quantitative analysis of CEUS for RILD. The horizontal axis represents the duration of the contrast agent(s). The vertical axis represents the strength achieved by the contrast agents. (A) Prior to exposure. The upper plot refers to a TIC obtained by quantitative analysis of CEUS. Prior to the exposure, the TIC presented as a single-peak curve with a steep upward rise and a slow decline after a short plateau. After the exposure, the rise and fall of TIC gradually slowed down with the aggravation of liver damage, with a gradually decreased peak. CEUS, contrast-enhanced ultrasound; RILD, radiation-induced liver damage; TIC, time-intensity curve.

presented as a single-peak curve with a steep upward rise and a slow decline after a short plateau. With the aggravation of liver damage, the rise and fall of TIC gradually slowed down and the peak value gradually decreased. Consequently, the TTP was gradually delayed and the Grad decreased slowly.
Results of TIC analysis. The TIC of the current study presented a single-peak curve with a steep upward rise and a slow decline after a short plateau. With the aggravation of liver damage, the rise and decline of TIC gradually slowed and the peak value gradually decreased. Consequently, the TTP was 
B
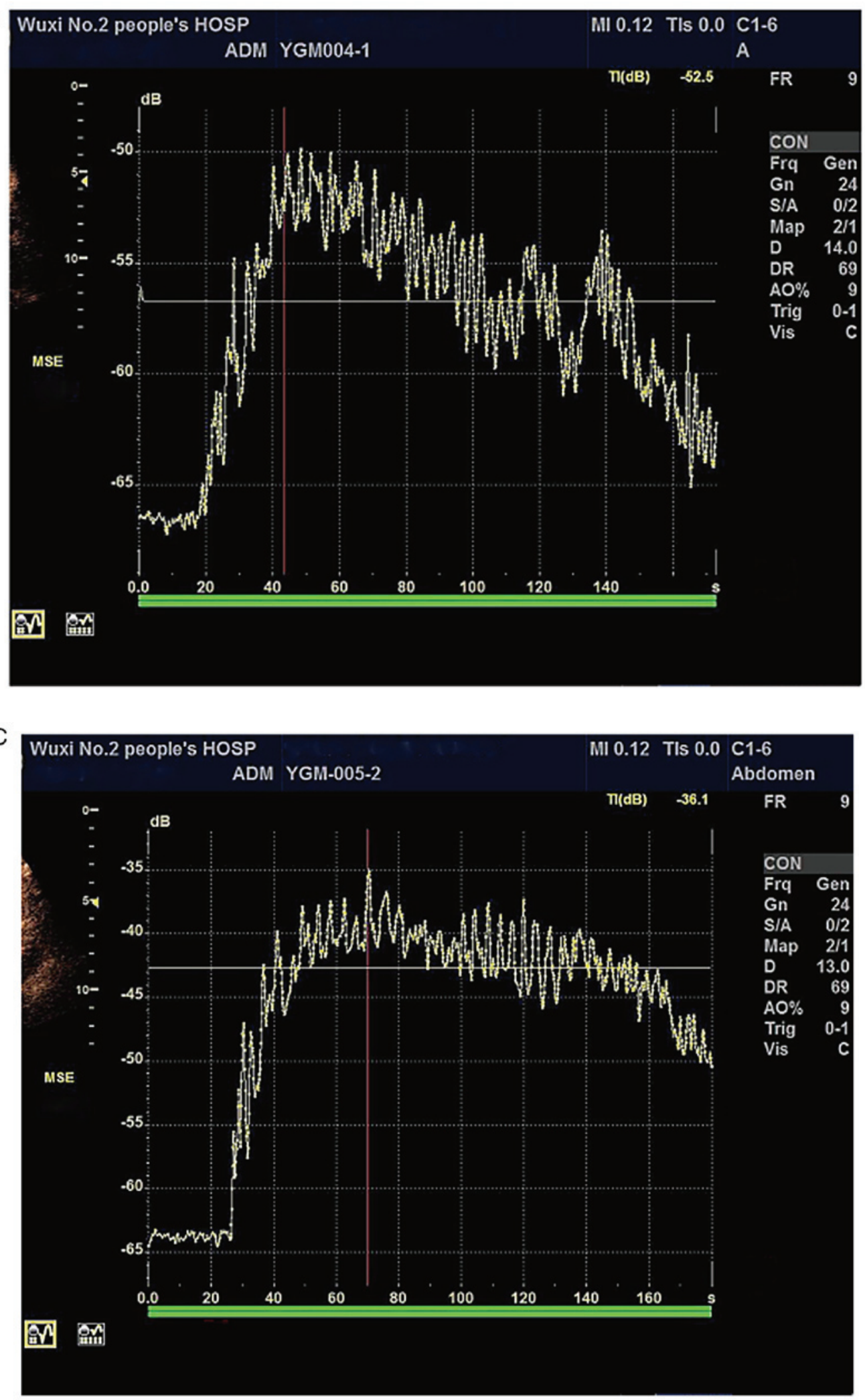

Figure 3. Continued. Images of quantitative analysis of CEUS for RILD. The horizontal axis represents the duration of the contrast agent(s). The vertical axis represents the strength achieved by the contrast agents. (B) Two weeks after exposure, (C) 4 weeks after exposure. The upper plot refers to a TIC obtained by quantitative analysis of CEUS. Prior to the exposure, the TIC presented as a single-peak curve with a steep upward rise and a slow decline after a short plateau. After the exposure, the rise and fall of TIC gradually slowed down with the aggravation of liver damage, with a gradually decreased peak. CEUS, contrast-enhanced ultrasound; RILD, radiation-induced liver damage; TIC, time-intensity curve.

gradually delayed and the Grad gradually decreased. In terms of the three parameters of the TIC, significant differences were identified in the Grad, TTP and AUC of the 2-, 3- or 4-week groups, as compared with those prior to exposure $(\mathrm{P}<0.05)$; the differences among the 2-,3- and 4-week groups were also statistically significant $(\mathrm{P}<0.05)$. Compared with that prior to 
Table I. Comparison of time-intensity curve parameters in quantitative analysis of contrast-enhanced ultrasonography for RILD.

\begin{tabular}{llll}
\hline Time-point & TTP $(\mathrm{sec})$ & Grad & AUC \\
\hline Pre-exposure & $14.371 \pm 2.491$ & $2.145 \pm 0.160$ & $3,220.75 \pm 128.381$ \\
2 weeks after exposure & $18.723 \pm 3.096^{\mathrm{a}}$ & $1.555 \pm 0.090^{\mathrm{a}}$ & $2,992.91 \pm 104.907^{\mathrm{a}}$ \\
3 weeks after exposure & $23.086 \pm 3.395^{\mathrm{a}, \mathrm{b}}$ & $1.038 \pm 0.088^{\mathrm{a}, \mathrm{b}}$ & $2,787.66 \pm 101.623^{\mathrm{a}, \mathrm{b}}$ \\
4 weeks after exposure & $30.174 \pm 2.017^{\mathrm{a}-\mathrm{c}}$ & $0.737 \pm 0.050^{\mathrm{a}-\mathrm{c}}$ & $3,248.78 \pm 87.264^{\mathrm{a}-\mathrm{c}}$ \\
\hline
\end{tabular}

${ }^{\mathrm{a}} \mathrm{P}<0.05$ compared with value prior to exposure; ${ }^{\mathrm{b}} \mathrm{P}<0.05$ compared with value 2 weeks after exposure; ${ }^{\mathrm{c}} \mathrm{P}<0.05$ compared with value at 3 weeks after exposure. AUC, area under the curve; Grad, gradient; TTP, time to peak.

Table II. AST and ALT levels in patients.

\begin{tabular}{lll}
\hline Groups & ALT (U/l) & AST (U/1) \\
\hline Pre-exposure & $24.44 \pm 11.68$ & $25.63 \pm 10.39$ \\
2 weeks after radiotherapy & $25.13 \pm 12.17$ & $28.52 \pm 12.94$ \\
3 weeks after radiotherapy & $30.30 \pm 10.20^{\text {a }}$ & $29.73 \pm 10.76^{\text {a }}$ \\
4 weeks after radiotherapy & $30.79 \pm 11.73^{\text {a }}$ & $31.26 \pm 13.55^{\text {a }}$
\end{tabular}

Data are expressed as the mean \pm SEM. ${ }^{\mathrm{a}} \mathrm{P}<0.05$. ALT, alanine aminotransamine; AST, aspartate transaminase.

exposure, the AUC decreased at 2 and 3 weeks, but increased at 4 weeks after the exposure (Table I).

AST and ALT results. Serological examination of 32 patients revealed that alanine aminotransamine (ALT) and aspartate transaminase (AST) increased gradually when the irradiation time was prolonged. There was a statistically significant difference in ALT and AST values of the 3- and 4-week groups, as compared with those prior to the exposure $(\mathrm{P}<0.05)$. There was no statistically significant difference between the 2 -week group and the pre-exposure group (Table II).

\section{Discussion}

In previous years, three-dimensional conformal intensity-modulated radiotherapy has been widely used for the treatment of abdominal malignant tumors; however, post-radioactive liver damage has become the most important factor affecting their radiation dose tolerance (7). Therefore, the early detection and diagnosis of acute RILD are of great significance for the restorative treatment of the liver. At present, the common examination methods for RILD are CT and MRI, but there is no specific imaging manifestation of early-stage RILD on CT and MRI $(3,4)$. CEUS displays the microcirculation of tissues and organs and the imaging effect is consistent with that of enhanced CT and MRI. To the best of our knowledge, no previous studies have assessed RILD in the hepatic microcirculation of the perfused liver, as previous study has only focused on liver tumors, cirrhosis and liver reperfusion injury (8).

In the present study, Grad, TTP and AUC were selected as the quantitative analysis parameters for CEUS. TTP is an index for the velocity of blood flowing in and out of the tissues, Grad reflects the average perfusion rate of the tissue and the AUC is associated to blood volume and flow velocity in the region. The results suggested that with the aggravation of liver injury, the liver parenchyma Grad exhibited a downward trend, the TTP gradually increased and the AUC decreased at 2 and 3 weeks, but increased at 4 weeks after the exposure. Based on the present results, it may be concluded that CEUS is able to reflect the changes of hepatic perfusion in patients with early-stage RILD. Timely and accurate assessment of the existence of RILD is of great significance for the selection of clinical treatment plans, disease monitoring and clinical prognosis.

Previous studies have indicated that typically, RILD pathologically manifests as a hepatic veno-occlusive disease (9). It is characterized by diffuse swelling and degeneration of liver cells, accompanied by necrosis and bleeding of liver cells, a significant increase of inflammatory cells in the portal area, as well as occlusion and congestion of the hepatic sinus and central venous lobule. Early lesions of RILD are mainly hepatic sinus congestion and inflammatory cell exudation following injury of sinusoidal endothelial cells. In the present study, CEUS was able to clearly reflect the changes in hepatic perfusion. The reasons may be as follows: i) In the early stage of acute RILD, the liver parenchyma undergoes fatty degeneration and the hepatocytes gradually swell, resulting in the compression and degeneration of the intrahepatic lumen structure, and a persistent decrease in blood flow perfusion in the liver parenchyma; ii) as liver injury progresses, infiltration of inflammatory cells in the liver parenchyma occurs, hepatocytes undergo degeneration and necrosis, and the inflammatory and necrotic tissues block the microcirculation pathway of the liver. At the same time, hepatic sinus congestion and inflammatory cell exudation caused by hepatic sinusoidal endothelial cell injury may cause narrowing and obstruction of the hepatic sinusoids, decreasing the blood flow into the hepatic sinusoids and resulting in a decrease in the blood volume in the hepatic sinusoids $(5,8,10)$; and iii) further aggravation of liver injury leads to fibrotic changes in the liver parenchyma. The increase in the intrahepatic fibrous structure compresses the peripheral sublobular vein of the liver, central vein and hepatic sinus, resulting in increased arteriovenous pressure, decreased blood flow in the microvessels and decreased total blood flow in the liver (11). The aforementioned causes lead to a decrease in the Grad of liver parenchyma and an increase in the TTP, which is gradually aggravated with the severity of RILD. 
The present study also indicated that the AUC gradually decreased with the severity of liver damage at 2 and 3 weeks, but increased at 4 weeks after the exposure, with significant differences between the three exposure and the one pre-exposure group. The reasons may be as follows: i) Hepatic sinus capillarization, which is similar to continuous capillaries and manifests as the damage of sinusoidal endothelial cells, reduction or disappearance of endothelial fenestrae and formation of a continuous basement membrane under the endothelium, may occur in the early stage of RILD. This change causes a disturbance in the exchange of substances and oxygen in the liver cells, eventually leading to hepatocyte atrophy and hepatic sinus collapse, a decrease in blood flow in the hepatic sinus and a gradual reduction of the AUC $(8,12)$; ii) as the liver damage intensifies, the hepatic sinus occlusion is aggravated, resulting in slow blood backflow, prolonged blood perfusion time in the liver at the late stage of the disease and thus an increase of the AUC; iii) intrahepatic cytokines (e.g. transforming growth factor $\beta 1$, connective tissue growth factor, platelet-derived growth factor) act as a driving factor to promote hepatic stellate cells to synthesize collagen fibers and accelerate their transformation into fibroblasts (13). The proliferation of fibroblasts and their connection with the surrounding fiber bundles lead to the formation of a fibrous septum. The blood vessels in the fibrous septum form a communication branch between the hepatic artery and portal and hepatic veins, which shunts the blood and causes an upturn in the overall blood flow in the liver. However, RILD progresses gradually; the cytokines are not significantly expressed in the early stage and their expression gradually increases after 2 weeks (11). In the present study, the AUC gradually decreased at 2 and 3 weeks after the exposure and significantly increased at 4 weeks.

In conclusion, quantitative analysis of CEUS may be used to accurately and objectively assess the changes and quantify the intrahepatic microcirculation perfusion in acute RILD. The TTP, Grad and AUC may be used as important reference indicators for the early diagnosis of acute RILD. Therefore, CEUS may be used to monitor liver hemodynamic changes in tumor patients following radiotherapy. For patients with abnormal TIC parameters, the corresponding liver protection treatment should be administered early and the radiation dose should be adjusted to delay or even prevent the occurrence and development of RILD. CEUS is expected to become a novel method for evaluating acute RILD.

\section{Acknowledgements}

Not applicable.

\section{Funding}

The current study was awarded two grants from the Wuxi Health Committee of Jiangsu Province (grant nos. YGM1126 and YGZXM1507) and one grant from the Science and Technology Development Fund of Nanjing Medical University (grant no. NMUB2018236).

\section{Availability of data and materials}

The datasets used and/or analysed during the current study are available from the corresponding author on reasonable request.

\section{Authors' contributions}

JF designed the study. TYX and SBC performed the research. YYG and PS analyzed the data. XC and YZC collected experimental data, created the database and wrote the manuscript. All authors read and approved the final manuscript.

\section{Ethics approval and consent to participate}

The study protocol was approved by the Institutional Review Board of the Second Hospital of Wuxi Affiliated to Nanjing Medical University (Wuxi, China) and all patients provided written informed consent.

\section{Patient consent for publication}

Not applicable.

\section{Competing interests}

The authors declare that they have no competing interests.

\section{References}

1. Pan CC, Kavanagh BD, Dawson LA, Li XA, Das SK, Miften M and Ten Haken RK: Radiation-associated liver injury. Int J Radiat Oncol Biol Phys 76 (Suppl 3): S94-S100, 2010.

2. Peixoto A, Pereira P, Bessa de Melo R and Macedo G: Radiation-induced liver disease secondary to adjuvant therapy for extra-hepatic cholangiocarcinoma. Dig Liver Dis 49: 227, 2017.

3. Jung J, Yoon SM, Cho B, Choi YE, Kwak J, Kim SY, Lee SW, Ahn SD, Choi EK and Kim JH: Hepatic reaction dose for parenchymal changes on Gd-EOB-DTPA-enhanced magnetic resonance images after stereotactic body radiation therapy for hepatocellular carcinoma. J Med Imaging Radiat Oncol 60: 96-101, 2015.

4. Guha $C$ and Kavanagh BD: Hepatic radiation toxicity: Avoidance and amelioration. Semin Radiat Oncol 21: 256-263, 2011.

5. Feng J, Chen SB, Wu SJ, Sun P, Xin TY and Chen YZ: Quantitative analysis of contrast-enhanced ultrasonography in acute radiation-induced liver injury: An animal model. Exp Ther Med 10: 1807-1811, 2015.

6. Edge SB and Compton CC: The American joint committee on cancer: The 7th edition of the AJCC cancer staging manual and the future of TNM. Ann Surg Oncol 17: 1471-1474, 2010.

7. Rahbari NN, Mehrabi A, Mollberg NM, Müller SA, Koch M, Büchler MW and Weitz J: Hepatocellular carcinoma: Current management and perspectives for the future. Ann Surg 253: 453-469, 2011.

8. Chapman TR, Kumarapeli AR, Nyflflot MJ, Bowen SR, Yeung RS, Vesselle HJ, Yeh MM and Apisarnthanarax S: Functional imaging of radiation liver injury in a liver metastasis patient: Imaging and pathologic correlation. J Gastrointest Oncol 6: E44-E47, 2015.

9. Da Silveira EB, Jeffers L and Schiff ER: Diagnostic laparoscopy in radiation-induced liver disease. Gastrointest Endosc 55: 432-434, 2002

10. Huang Y, Chen SW, Fan CC, Ting LL, Kuo CC and Chiou JF: Clinical parameters for predicting radiation-induced liver disease after intrahepatic reirradiation for hepatocellular carcinoma. Radiat Oncol 11: 89, 2016.

11. Xia H, Deng L and Jin Y: An experimental study on acute radiation-induced liver damage and its protection in rats. J Cancer Control Treat 24: 9-13, 2011.

12. Benson R, Madan R, Kilambi R and Chander S: Radiation induced liver disease: A clinical update. J Egypt Natl Canc Inst 28: 7-11, 2016.

13. Lee IJ, Seong J, Shim SJ and Han KH: Radiotherapeutic parameters predictive of liver complications induced by liver tumor radiotherapy. Int J Radiat Oncol Biol Phys 73: 154-158, 2009. 